\title{
TDOA-Based and RSSI-Based Underground Wireless Positioning Methods and Performance Analysis
}

\author{
Guofang Dong ${ }^{1,2,3,4}$ and Bin Yang ${ }^{1,2,3}$ \\ ${ }^{1}$ National Engineering Laboratory For Vacuum Metallurgy, Kunming University \\ of Science And Technology, Kunming 650093, China \\ ${ }^{2}$ Key Laboratory for Nonferrous Vacuum Metallurgy of Yunnan Province, \\ Kunming University of Science and Technology, Kunming 650093, China \\ ${ }^{3}$ School of Metallurgy and Energy Engineering, Kunming University of Science \\ and Technology, Kunming 650093, China \\ ${ }^{4}$ School of Electrical and Information Technology, Yunnan Nationalities \\ University, Kunming 650031, China \\ dongguofangl@163.com
}

\begin{abstract}
Wireless location has become one of the important technologies of wireless sensor network application. Due to the underground wireless location affected by various factors, the positioning system cannot meet the actual demand. In this paper, Time Difference of Arrival (TDOA) and Received Signal Strength Indicator (RSSI) location technologies have been investigated, then various impact factors on the TDOA and RSSI location systems have been discussed, multipath channel, different shapes of the roadway on the propagation loss. In order to reduce the error, estimation error has been correlated and compensated. Simulations show that the proposed system consistently outperforms other existing localization methods and can achieve higher accuracy.
\end{abstract}

Keywords: TDOA, RSSI, Underground Location

\section{Introduction}

Localization is one of the key techniques in wireless sensor network which have displaced the well-established and widely-deployed wired communication networks. As a critical role in many applications, Localization is getting more and more attention. The positions of unknown nodes play a critical role in many WSNs applications, for examples: Resource tracking, proximity-based notification.

To implement a localization system, RSSI, time of arrival (ToA)-based, and TDoAbased localization systems are widely used because these systems can be readily implemented on the existing wireless infrastructure. However, various forms of interference always occur with advanced communication technologies, such as low transmission power, propagation attenuation $[1,2]$. Furthermore, the ITU-R Report SM.2211 presents the advantage and disadvantage of TDOA-based technologies [3]. The transmitter and receiver must be synchronized precisely when using TOA location technology. The AOA of location technology is limited by Non-Line-of-sight (NLOS) and multipath effects. Recently, the integrated TDOA/AOA location systems reduce various positioning errors and improve the location accuracy and extend the location coverage area [4-7].

Luo evaluates the accuracy of several RSSI-based localization techniques and compares them in a real environment. The results indicate that The simple MinMax algorithm has better performance than other algorithms, with average errors as low as 1.2 $\mathrm{m}$ [8]. Xu proposed an improved RSSI underground positioning algorithm which is based 
on wireless sensor networks. The system improved precision of node positioning by iteration computation and solved potential conflicts during data transfer [9]. Zhu and Yi proposed an underground mine positioning method is described for accurate localization of a sensor in a network of known beacons. The sensor measures the range using an UWB signal and uses statistical inference to correct for the error due to multipath and NLOS [10]. Li and Liu proposed a weighted centroid localization algorithm in underground mine wireless sensor networks based on RSSI algorithm. By considering the underground wireless signal transmission attenuation model, simulation results show that new algorithm improves the localization accuracy [11].

Although many researchers have achieved a lots in localization algorithms, especially for the range-based location. Due to the underground wireless location affected by various factors, the positioning system is still very immature and system accuracy cannot meet the actual demand. In this paper, firstly TDOA and RSSI location technologies are investigated and the corresponding error various impact factors and expressions are discussed and derived, multipath channel, different shapes of the roadway on the propagation loss. In order to reduce the error, estimation error has been correlated and compensated. Simulations show that the proposed system consistently outperforms other existing localization methods and can achieve higher accuracy.

\section{Ranging Model}

\subsection{TDOA}

TDOA is one of TOF (the time of flight) ranging method-based localization technology. Due to its high accuracy, it has been widespread concern. The basic idea of TDOA is to measure the arrival time difference between the transmitting base station and the receiving base station, then the distance difference to a different base station is calculated based on the time difference. The mobile station to the difference in distance between any two base stations form a hyperbola on which the mobile station is located in a curve. Figure 1 illustrates the basic TODA positioning method.

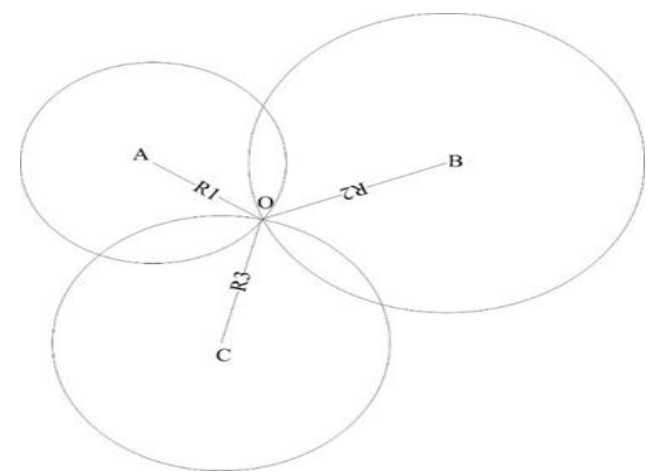

Figure 1. TDOA Positioning Method

Because of interferometry techniques, a reference node with two antennas can be adopted, the two antennas A1 and A2 transmit the same signal from a unique source. Assume Line-of-sight propagation between reference node and mobile node, $\mathrm{d} 1$ denotes the distance between mobile node and $\mathrm{A} 1, \mathrm{~d} 2$ denotes the distance between mobile node and A2. The delays of propagation are $t_{1}=d_{1} / c$ and $t_{2}=d_{2} / c$, with $\mathrm{c}$ the speed of signal. $\mathrm{A} 1$ and $\mathrm{A} 2$ transmit the same signals with a sample rate frequency Fs, $\mathrm{N}$ sub-carriers and $\mathrm{M}$ data sub-carriers, but with a delay of $\mathrm{Tp}$ which varies the delay of the signal in a predetermined manner, $x(t)$ denotes the baseband complex envelope signal and, $x R F(t)$ denotes the transmitted signal, following equations are obtained: 


$$
\begin{array}{r}
x(\mathrm{t})=\sum_{k=-N / 2}^{N / 2-1} C_{k} e^{i 2 \pi k F_{s} t / N} \\
x_{R F}(\mathrm{t})=x(\mathrm{t}) e^{i 2 \pi F_{R F} t}
\end{array}
$$

ck are complex coefficients, with $\mathrm{k}$ the carrier index and FRF the RF frequency. Assuming that the signal through the multipath channel $\mathrm{p}(\mathrm{t})$,then

$$
Z(\mathrm{t})=\sum_{m=-N / 2}^{N / 2-1} p(\mathrm{~m}) \mathrm{x}(\mathrm{t}-\mathrm{m})
$$

$\mathrm{P}(\mathrm{m})$ is the propagation attenuation coefficient of the $\mathrm{m}$ th path。 time invariant channel impulse responses in the CE domain for the two different delays of propagation,t1 and $\mathrm{t} 2$, are:

$$
\begin{array}{r}
h_{1}(\mathrm{t})=\mathrm{h}_{1} e^{-i 2 \pi F_{R F} t_{1}} \delta\left(\mathrm{t}-\mathrm{t}_{1}\right) \\
h_{2}(\mathrm{t})=\mathrm{h}_{2} e^{-i 2 \pi F_{R F}\left(\mathrm{t}_{2}+\mathrm{t}_{\mathrm{p}}\right)} \delta\left(\mathrm{t}-\mathrm{t}_{2}-\mathrm{t}_{\mathrm{p}}\right)
\end{array}
$$

where $\&$ is the Dirac function. Suppose the channel noise $v(n)$, the received signal at $\mathrm{MD}, \mathrm{y}(\mathrm{t})=\mathrm{x}(\mathrm{t}) \otimes \mathrm{h}(\mathrm{t})$ in complex envelope domain is equal to:

$$
y(\mathrm{t})=h_{1} x\left(\mathrm{t}-\mathrm{t}_{1}\right) e^{-i 2 \pi F_{R F} t_{1}}+h_{2} x\left(\mathrm{t}-\mathrm{t}_{2}-\mathrm{t}_{\mathrm{p}}\right) e^{-i 2 \pi F_{R F}\left(\mathrm{t}_{2}+\mathrm{t}_{p}\right)}+v(\mathrm{n})
$$

\subsection{RSSI}

The reference node broadcasts signal to the mobile node, then the mobile node can estimate the distance between it and reference node on the basis of the strength of the signals it receive. Assume reference node located at (xi, yi) and a mobile node located at $(x, y)$.The average received power from reference node $\mathrm{i}$ at mobile node $\mathrm{j}$, in $\mathrm{dB}$, can be expressed as

$$
p_{i j}=p_{0 i}-10 \beta \log \left(\frac{d\left(\mathrm{x}_{\mathrm{i}}, \mathrm{x}_{\mathrm{j}}\right)}{d_{0}}\right)+\mathrm{n}_{\mathrm{ij}}
$$

where $\mathrm{d} 0$ is a reference distance, $\mathrm{P} 0 \mathrm{i}$ denotes the power at distance $\mathrm{d} 0, \beta$ is a path-loss exponent [12], and nij is a zero-mean Gaussian random variable with standard deviation $\sigma$ [13]. As can be seen, the model is nonlinearly dependent on the position of the transmitter i. Here,

$$
f\left(\mathrm{x}_{\mathrm{i}}, \mathrm{x}_{\mathrm{j}}\right)=p_{0 i}-10 \beta \log \left(\frac{\|\left(\mathrm{x}_{\mathrm{i}}, \mathrm{x}_{\mathrm{j}} \|\right.}{d_{0}}\right)
$$

If POi and $\beta$ are known, the maximum likelihood estimate of the distance between node $\mathrm{i}$ and $\mathrm{j}$ can be computed as

$$
\hat{d}_{i j}=\mathrm{d}_{0} 10 \frac{p_{0 i}-p_{i j}}{10 \beta}
$$

Obviously, the distance estimate ${ }^{\hat{d}_{i j}}$ is biased and an unbiased estimate for the distance can be computed as [12].

$$
\hat{d}_{i j}{ }^{\prime}=d_{0} 10^{\frac{p_{0 i}-p_{i j}}{10 \beta} e^{-\frac{10 \beta}{\sigma i_{j} \ln 10}}}
$$


The Cramer-Rao lower-bound for the variance of unbiased distance estimator based on RSSI measurements can be obtained as [14]

$$
E\left(\hat{d}_{i j}-E\left(\hat{d}_{i j}\right)\right)^{2} \geq\left(\frac{\sigma_{i j} d\left(\mathrm{x}_{\mathrm{i}}, \mathrm{x}_{\mathrm{j}}\right) \ln 10}{10 \beta}\right)^{2}
$$

It is observed that the estimate accuracy is closely related with the distance $\mathrm{d}(\mathrm{xi}, \mathrm{xj})$, the standard deviation ${ }^{\sigma_{i j}}$ of measurement noise and the path-loss exponent ${ }^{\beta}$.

\subsection{Electromagnetic Propagation Attenuation}

Kim studied electromagnetic propagation in tunnels, and proved electromagnetic propagation related with signal frequency, tunnel cross sectional dimensions and shape, curves in the tunnel, surface roughness [15]. Tunnel cross section has an important impact on electromagnetic propagation attenuation rate compare to other impact factors. Yamaguchi proposed a point-matching method for attenuation constants of Ultra High Frequency (UHF) radio waves in arched tunnels [16]. Zhang studied signal propagation characteristics and proposed signal attenuation calculating expression for different tunnels, like circular, rectangular, arched and ellipse tunnels $[17,18]$.
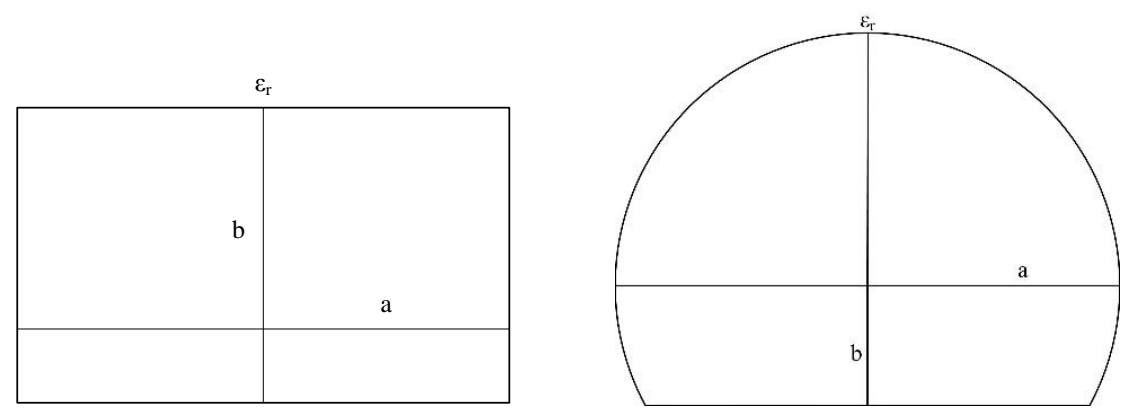

Figure 2. Rectangular and Circular Cross Sections of the Tunnels

For circular and rectangular tunnel cross section shown in Figure 2, the equations for radio signal attenuation in $\mathrm{dB} / \mathrm{m}$ are proposed in [17] separately:

$$
\begin{gathered}
\alpha=5.09 \lambda^{2}\left\lfloor\frac{\varepsilon_{r}}{\alpha^{3} \sqrt{\varepsilon_{r}-1}}+\frac{1}{b^{3} \sqrt{\varepsilon_{r}-1}}\right\rfloor \\
\alpha=4.343 \lambda^{2}\left\lfloor\frac{\varepsilon_{r}}{\alpha^{3} \sqrt{\varepsilon_{r}-1}}+\frac{1}{b^{3} \sqrt{\varepsilon_{r}-1}}\right\rfloor
\end{gathered}
$$

In the formula, $\mathrm{a}$ is the maximum tunnel width, $\mathrm{b}$ is the maximum height, and $\varepsilon_{r}$ is the relative dielectric constant of the tunnel walls and floors. For reliable communication it is preferable to install additional antennas and thus maintain the conditions of direct visibility (LOS) between adjacent antennas [19]. So the average received power pij, signal attenuation power pa can be expressed as

$$
p_{i j}=p_{0 i}-10 \beta \log \left(\frac{d\left(\mathrm{x}_{\mathrm{i}}, \mathrm{x}_{\mathrm{j}}\right)}{d_{0}}\right)-P_{a}+\mathrm{n}_{\mathrm{ij}}
$$




$$
P_{a}=5.09 \lambda^{2}\left\lceil\frac{\varepsilon_{r}}{\alpha^{3} \sqrt{\varepsilon_{r}-1}}+\frac{1}{b^{3} \sqrt{\varepsilon_{r}-1}}\right\rfloor d
$$

Where $d=\left|d\left(\mathrm{x}_{\mathrm{i}}, \mathrm{x}_{\mathrm{j}}\right)-d_{0}\right|$

\section{Location Estimation}

The measurement between mobile node $\mathrm{i}$ and reference node $\mathrm{j}$ is defined as a function which is given by $m_{i j}=f\left(\mathrm{x}_{\mathrm{i}}, \mathrm{a}_{\mathrm{j}}\right)+\varepsilon_{i j}$, where $f\left(\mathrm{x}_{\mathrm{i}}, \mathrm{a}_{\mathrm{j}}\right)$ is a deterministic function that defines a type of error-free measurement between node at position ${ }^{x_{i}}$ and node at position $\mathrm{a}_{j}$, and ${ }^{\varepsilon_{i j}}$ is the measurement error. The measurement errors ${ }^{\varepsilon_{i j}}$ may have any probability density function. In this case, the measurement error of TDOA caused in the line of sight is obeyed by Gaussian distribution. Measurements can be collected at reference nodes, mobile nodes, or both reference and mobile nodes.

\subsection{Maximam Likelihood}

Assume that measurement errors are independent and identically distributed random variables. Let the measurements' probability density function is $p_{M_{i j}}\left(\mathrm{~m}_{\mathrm{ij}} ; \mathrm{X}\right)$ in $m_{i j}=f\left(\mathrm{x}_{\mathrm{i}}, \mathrm{a}_{\mathrm{j}}\right)+\varepsilon_{i j}$, where $X=\left[\mathrm{x}_{1}, \ldots, \mathrm{x}_{\mathrm{M}}\right]^{\mathrm{T}} \in \mathrm{R}^{2 \times \mathrm{M}}$.

For independent and identically distributed Gaussian measurement errors, the maximum likelihood estimator can be computed as

$$
\hat{X}=\underset{X \in R^{2 \times M}}{\arg \min } \sum_{i=1}^{M}\left(\sum_{j \in A_{i}} \frac{1}{\sigma^{2}}\left(\mathrm{~m}_{\mathrm{ij}}-\mathrm{f}\left(\mathrm{x}_{\mathrm{i}}, \mathrm{a}_{\mathrm{j}}\right)\right)^{2}\right)
$$

where ${ }^{2}{ }_{i j}$ is the variance of measurement errors.

For the TOA approach distance measurements, equation (16) can be written as

$$
\hat{X}=\arg \underset{X \in R^{2 \times M}}{\min } \sum_{i=1}^{M}\left(\sum_{j \in A_{i}} \frac{1}{\sigma_{i j}^{2}}\left(\hat{d_{i j}}-\left\|\mathrm{x}_{\mathrm{i}}-\mathrm{x}_{\mathrm{j}}\right\|\right)^{2}\right)
$$

\subsection{Nonlinear Least Squares}

In position estimation, Nonlinear Least Squares(NLS) algorithm tries to minimize the following cost function:

$$
\hat{X}=\arg \underset{X \in R^{2 \times M}}{\min } \sum_{i=1}^{M}\left(\sum_{j \in A_{i}}\left(\mathrm{~m}_{\mathrm{ij}}-\mathrm{f}\left(\mathrm{x}_{\mathrm{i}}, \mathrm{a}_{\mathrm{j}}\right)\right)^{2}\right)
$$

When the variances of measurement errors are available, the NLS can be formulated as a weighted nonlinear least squares :

$$
\hat{X}=\arg \underset{X \in R^{2 \times M}}{\min } \sum_{i=1}^{M}\left(\sum_{j \in A_{i}} \frac{1}{\sigma^{2}}\left(\mathrm{~m}_{i j}-\mathrm{f}\left(\mathrm{x}_{\mathrm{i}}, \mathrm{a}_{\mathrm{j}}\right)\right)^{2}\right)
$$

If measurement errors are independent and identically distributed Gaussian measurement errors, the weighted nonlinear least squares solution is same as the ML solution in the range-based positioning. 


\section{Simulation Results}

\subsection{Simulation Environment}

Mine galleries run twenty kilometers in Huize $\mathrm{Pb}-\mathrm{Zn}$ mine, part of representative galleries can be drawn as Figure 2. The location system is installed in the marked rectangular area of Figure 3.

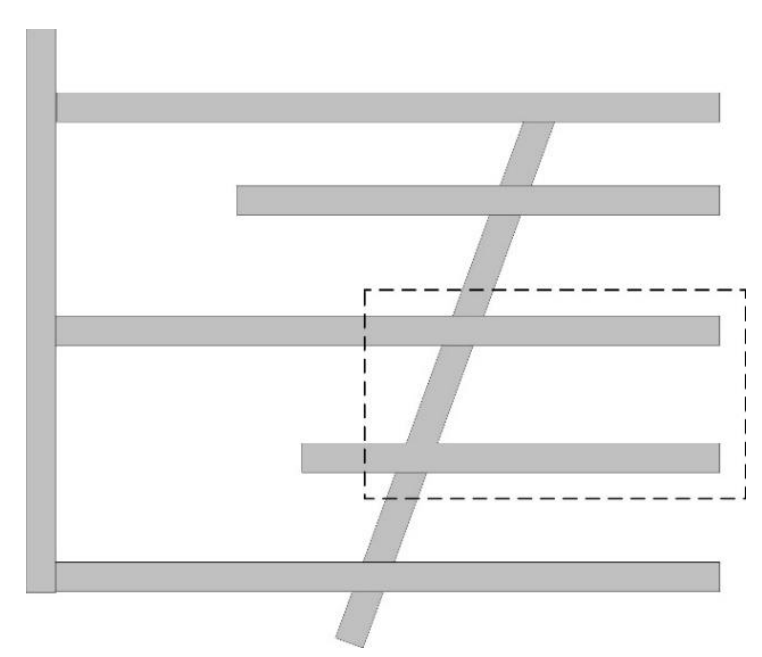

Figure 3. Representative Mine Galleries in Huize Pb-Zn Mine

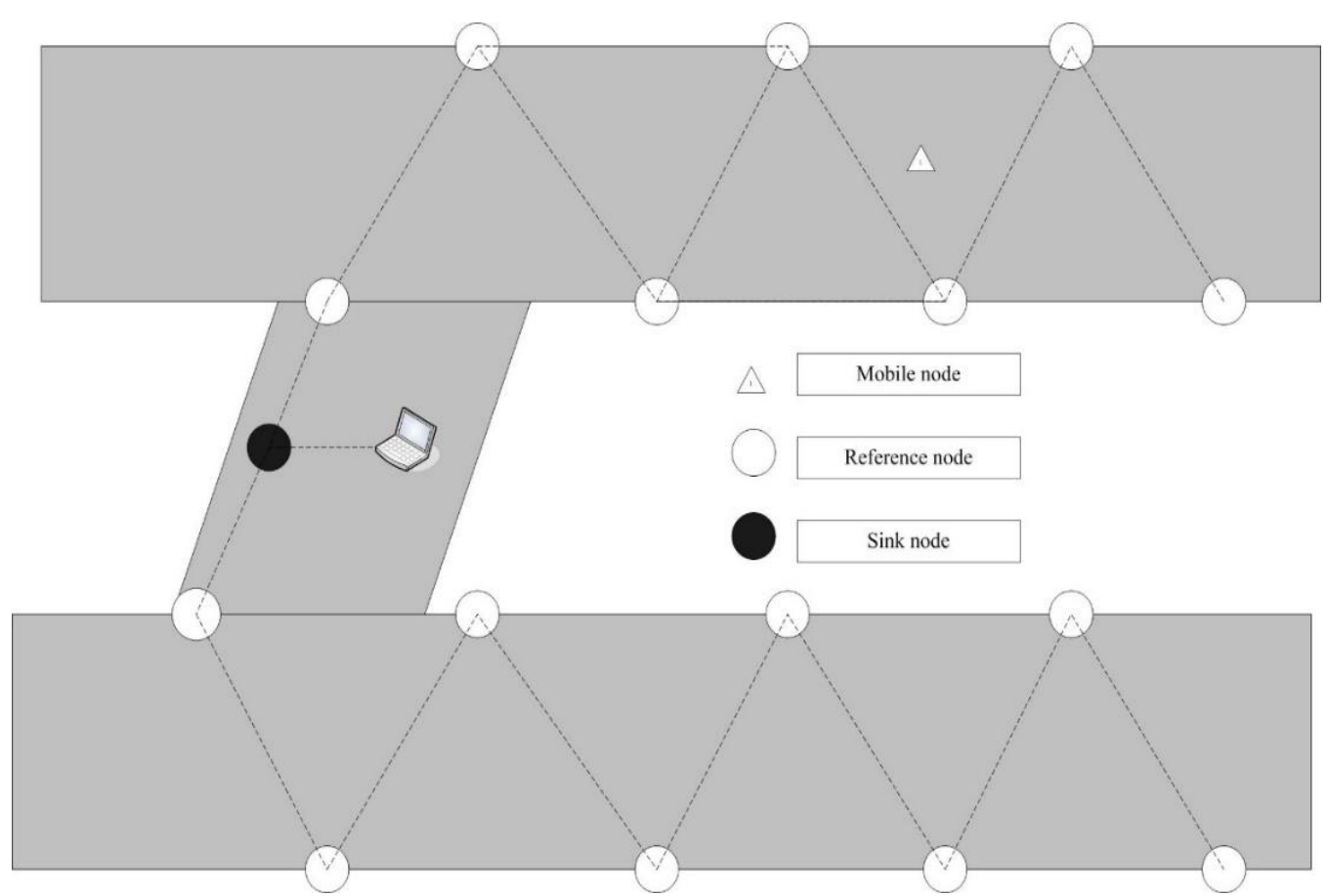

Figure 4. The Location System in the Selected Area

In this paper, the localization system consists of a surveillance PC, a sink node, fifteen reference nodes and one or more mobile nodes. One of the well known topology in location is the triangulation, so reference nodes are arranged as Figure 4. The whole structure of the system is shown in Figure 4. 
The wireless sensor networks in mine galleries is a ZigBee network and the reference node is responsible for establishing the network. The reference node collects data of mobile node in mine galleries environment. The reference node also acts as a ZigBee Router to the sink node and communicates with adjacent reference nodes. The sink node collects data from reference nodes and sends data to surveillance PC. The surveillance PC is responsible for localization data management and computation.

\subsection{Experimental Results}

To show the superiority of TDOA-based localization over RSSI-based localization in mine tunnel environment, a contrast measuring experiment was carried out in a point to point way, using TDOA and RSSI ranging methods respectively. Locations were estimated at 15 test points. At each known position, 20 times of TDOA ranging and 20 times of RSSI ranging were performed. The distance between mobile node and one of the reference node is 5 to 75 meters.

The energy of the radio signal distorts seriously because of the multi-path and attenuation effect. Figures 5 shows the standard deviation of the measuring results from TDOA-based measuring and RSSI-based measuring. As can be seen from the result, the distance estimation errors according to RSSI increase significantly and the standard deviation of TDOA measurements increases with the increase of distance. As a conclusion, RSSI is not suitable for distance estimation in underground tunnel environment. TDOA shows better performance than RSSI in standard deviation and the ranging error increasing with the increase of distance. Furthermore, most of the ranging errors are less than 4 meters in TDOA-based measuring.

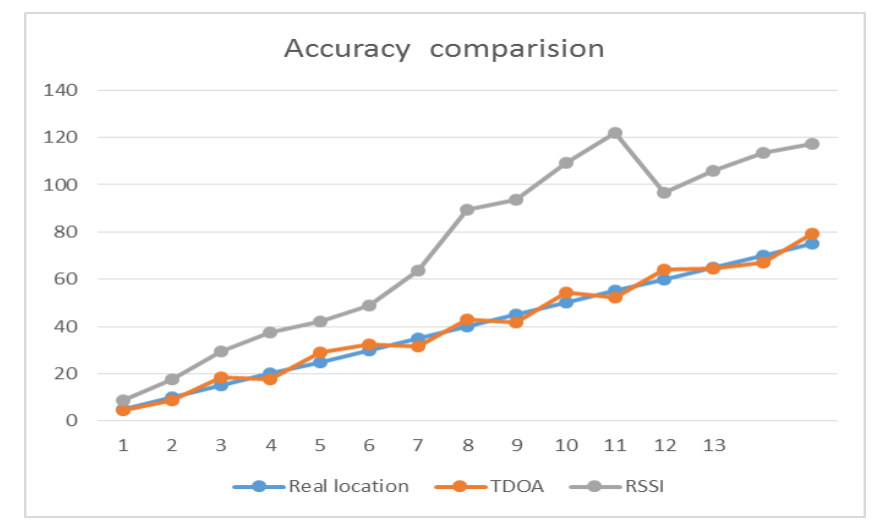

Figure 5. The Accuracy Comparison of TDOA and RSSI Location

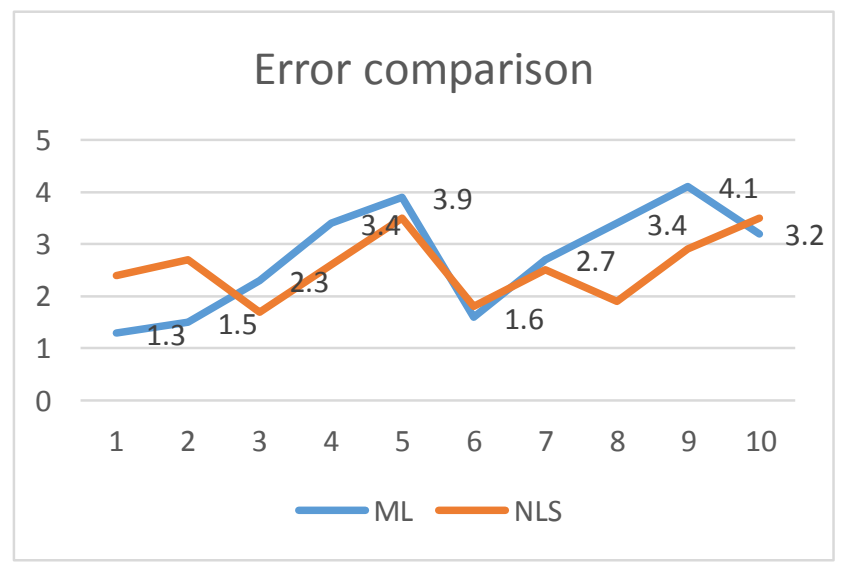

Figure 6. The Average Location Errors Comparison of ML, NLS 
Other existing algorithm, NLS was implemented as well in the same experiment environment. Both ML and NLS algorithms were tested under the same condition. Figure 6 showed the average localization errors of the two algorithms at each test point. Two curves had the similar trend, which means that large range error degraded the performance of the two localization algorithms, but the influence to NLS was smaller than the ML.

\section{Conclusions}

In this paper, a wireless sensor networks was arranged for location in mine galleries. The proposed wireless sensor networks is based on the triangular position of beacons. The probability error and effects of the received signal in mine galleries are analyzed, then estimation of location of the mobile node is derived. According to our analyses and simulations, the proposed system consistently shows accurate estimation. In the future research, underground wireless positioning model need to full study to reduce the error of distance measurement, how to further improve the positioning accuracy in the complex underground wireless environment is the direction of future research.

\section{Acknowledgments}

This study was supported by the Application Foundation Research Project of Yunnan Science and Technology Department (No. 2011FZ168), the National Science foundation of China (No. 61461056).

\section{References}

[1] Y. C. Liang, A. R. Leyman and B. H. Soong, "Multipath Time Delay Estimation Using Higher Ordesr Statistics", Proceedings of the IEEE Signal Processing Workshop on Higher-Order Statistics, Banff, Canada, (1997) July, pp. 9-13.

[2] A. Hrovat, G. Kandus and T. Javornik, "A Survey of Radio Propagation Modeling for Tunnels", IEEE Communications Surveys \& Tutorials, vol.16, no.2, (2014)May, pp.658-669.

[3] ITU-R SM. 2211-1, "Comparison of Time-Difference-of-Arrival and Angle-of-Arrival Methods of Signal Geolocation", (2014)June.

[4] W.H. Zhuang and Li C., "Hybrid TDOA/AOA Mobile User Location for Wideband CDMA Cellular Systems", IEEE Transactions on Wireless Communications, vol.1,no.3, (2002)August, pp. 439-447.

[5] J. Xu, M. Ma. and C.L. Law, "Position Estimation Using Ultra Wideband Time Difference Of Arrival Measurements", IET Science, Measurement \& Technology, vol.2. No.1, (2007) December, pp.53-58.

[6] S. Bohanudin, M. Ismail and H. Hussain, "Simulation Model And Location Accuracy For Observed Time Difference Of Arrival (OTDOA) Positioning Technique In Third Generation System”, 2010 IEEE Student Conference on Research and Development (SCOReD), Putrajaya, Malaysia, (2010) December, pp. 63-66.

[7] Q. Qineti, ARUP and TRL, Ofcom AMS Final Report, London, United Kingdom, (2006) July.

[8] X. Luo, W. J. O’Brien and C. L. Julien, “Comparative Evaluation Of Received Signal-Strength Index (RSSI) Based Indoor Localization Techniques For Construction Jobsites", Advanced Engineering Informatics, vol. 25, no. 2, (2011) April, pp. 355-363.

[9] Y. Xu, Z. Sun and C. Li, "The Design and Realization of Underground Location System Based on ZigBee Technology", Proceedings of the International Conference on Green Communications and Networks, Lecture Notes in Electrical Engineering, vol. 113, (2012), pp. 511-518.

[10] D. Zhu and K. Yi, "Localization Technology Research Based on Particle Filter in Underground Mines", Journal of Computational Information Systems, vol. 9, no. 8, (2013), pp. 3011-3018.

[11] L. Jian and L. He-Ping, "A New Weighted Centroid Localization Algorithm In Coal Mine Wireless Sensor Networks", 2011 3rd International Conference on Computer Research and Development (ICCRD), vol. 3, (2011) March, pp. 106-109.

[12] N. Patwari, "Location Estimation In Sensor Networks", Ph.D. dissertation, University of Michigan, Ann Arbor, (2005)

[13] A. F. Molisch, "Wireless Communications", New York, NY: John Wiley\& Sons, (2010).

[14] S. Gezici, "A Survey On Wireless Position Estimation", Wireless Personal Communications (Special Issue on Towards Global and Seamless Personal Navigation), vol. 44, no. 3, (2008) February, pp. 263282. 
[15] Y. Kim, M. Jung and B. Lee, "Analysis Of Radio Wave Propagation Characteristics in Rectangular Road Tunnel At 800 Mhz And 2.4 Ghz", Antennas and Propagation Society International Symposium, vol. 3, (2003), pp. 1016-1019.

[16] Y. Yamaguchi, T. Abe, Toshio and J. Chiba, "Attenuation Constants of UHF Radio Waves in Arched Tunnels", IEEE Transactions on Microwave Theory and Techniques, vol. 33, no. 8, (1985), pp. 714-718.

[17] Z. Changsen and M. Yan, "Effects of Cross Section Of Mine Tunnel On The Propagation Characteristics Of UHF Radio Wave", 7th International Symposium on Antennas, Propagation EM Theory, (2006), pp. $1-5$.

[18] Z. Chang-sen and G. Li-fang, "Research on Propagation Characteristics of Electromagnetic Wave in Tunnels with Arbitrary Cross Sections", 2nd International Conference on Future Computer and Communication (ICFCC), vol. 1, (2010), pp. V1-22-V1-25.

[19] X. Yang and Y. Lu, "Research on Propagation Characteristics of Millimeter Wave in Tunnels", International Journal of Infrared and Millimeter Waves, vol. 28, no. 10, (2007), pp. 901-909.

\section{Authors}

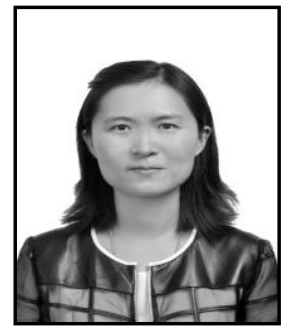

Guofang Dong, she was born in China in 1979. He received his B.E. and M.E. from Chongqing University of Posts and Telecommunications, China, in 2001 and 2007, respectively. She has been working towards her Ph.D. in Kunming University of Science and Technology. Her current research interests include wireless sensor networks, information security.

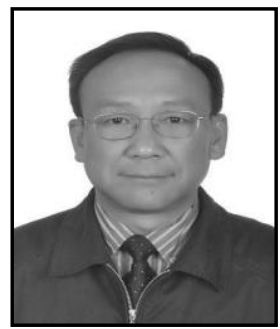

Bin Yang, he was born in China in 1965. He received his B.E. from Northeastern University, China, in 1987. He received his M.E. from Kunming University of Science and Technology, China, in 1990. He received his Ph.D. from Kunming University of Science and Technology, China, in 1998. Now he is the professor of Kunming University of Science and Technology. 
International Journal of Future Generation Communication and Networking Vol. 8, No. 5 (2015) 